\title{
Caracterização dos componentes internos e qualidade dos ovos de perdiz brasileira (Rhynchotus rufescens) durante 28 dias de armazenamento
}

\author{
Characterization of internal components and quality of red-winged tinamou (Rhynchotus rufescens)
}

eggs throughout $28 \mathrm{~d}$ storage time

Caracterización de los componentes internos y calidad de huevos de la perdiz brasileña

(Rhynchotus rufescens) durante 28 días de almacenamiento

Recebido: 21/12/2020 | Revisado: 29/12/2020 | Aceito: 30/12/2020 | Publicado: 04/01/2021

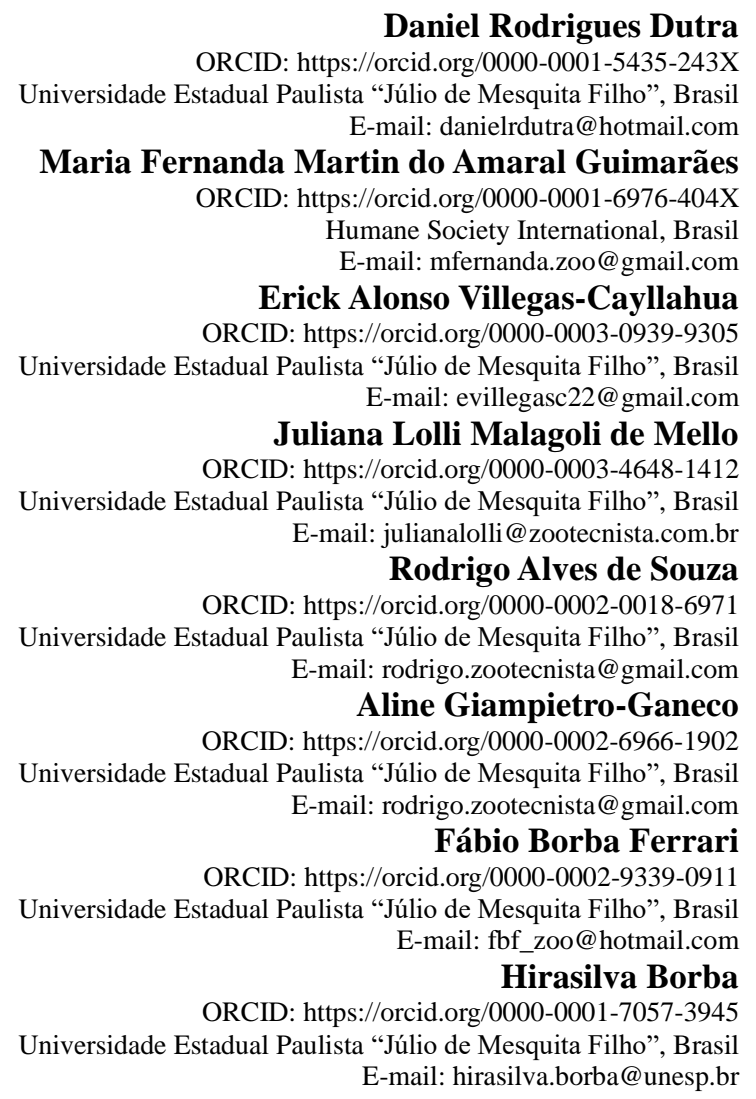

\section{Resumo}

Com o objetivo de definir as características internas e verificar o efeito do armazenamento sobre os ovos de perdizes brasileiras (Rhynchotus rufescens), foram avaliados um total de 50 ovos, distribuídos em delineamento inteiramente casualizado e mantidos em temperatura ambiente por 28 dias. Foram avaliados o peso médio dos ovos, altura de albúmen, largura e altura de gema, unidade Haugh, índice de gema, $\mathrm{pH}$ dos componentes internos e coloração de gema crua. A coloração da gema foi determinada pelo método direto de caracterização cromática (parâmetros de luminosidade (L) e cromaticidade $\left(\mathrm{a}^{*}\right)\left(\mathrm{b}^{*}\right)$ ). As avaliações ocorreram nos dias $0,10,15,21$ e 28 após a postura. Ao longo do período experimental, houve redução $(\mathrm{p}<0,05)$ da altura de albúmen e da unidade Haugh, elevação $(\mathrm{p}<0,05)$ do $\mathrm{pH}$ de albúmen, diminuição $(\mathrm{p}<0,05)$ da intensidade de vermelho $\left(\mathrm{a}^{*}\right)$ e aumento $(\mathrm{p}<0,05)$ da intensidade de amarelo ( $b *$ ) na gema. O valor médio da unidade Haugh diminuiu de 84,74 para 41,59, o tamanho do albúmen reduziu em média 4,37 mm e tornou-se, ainda mais alcalino ( $\mathrm{pH}$ final 9,58) e a gema apresentou uma coloração mais amarelada, com aumento de 11,51 índice b* e redução de $-1,01$ índice $a^{*}$, ao final dos 28 dias de armazenamento. Os demais parâmetros de qualidade da gema não foram influenciados $(p>0,05)$ pelo tempo. Conclui-se, portanto, que os ovos de perdizes brasileiras possuem características internas com grande potencial de exploração comercial. 
Entretanto, 28 dias de armazenamento em temperatura ambiente é suficiente para afetar a qualidade do albúmen e a coloração da gema.

Palavras-chave: Albúmen; Gema; Índice de gema; pH; Unidade Haugh.

\begin{abstract}
With the objective of characterizing the inner traits of red-winged tinamou (Rhynchotus rufescens) eggs at reproductive phase and evaluate the effects of the storage on quality indicators, a total of 50 eggs were distributed in a completely randomized design and kept at room temperature for 28 experimental days. Average egg weight, Haugh unit, yolk index, width and height of yolk, albumen height, $\mathrm{pH}$ of internal components and yolk color were evaluated. Yolk color scores were determined by direct method of chromatic traits (parameters of lightness (L) and chromaticity $(\mathrm{a} *)(\mathrm{b} *))$. Assessments were made on days 0, 10, 15, 21 and 28 after laying. During the experimental period there was a reduction $(\mathrm{p}<0.05)$ of the Haugh unit and albumen height, albumen $\mathrm{pH}$ elevation $(\mathrm{p}<0.05)$, reduction $(\mathrm{p}<0.05)$ of redness $\left(\mathrm{a}^{*}\right)$ and increased $(\mathrm{p}<0.05)$ yellowness $\left(\mathrm{b}^{*}\right)$ in the yolk. Throughout the storage time, the average Haugh unit value declined from 84.74 to 41.59 , albumen reduced average size into $4.37 \mathrm{~mm}$ and became more alkaline (final $\mathrm{pH}$ 9.58). The yolk presented itself yellowish, with an increase of $11.51 \mathrm{~b}^{*}$ index and a reduction of $-1.01 \mathrm{a}^{*}$ index. The other parameters of yolk quality were not affected $(\mathrm{p}>0.05)$ by the storage time. It is concluded, therefore, that red-winged tinamous eggs have internal characteristics with great potential for commercial exploitation. However, 28 days of storage at room temperature is enough to affect the albumen quality and yolk color.
\end{abstract}

Keywords: Albumen; Haugh unit; pH; Yolk; Yolk index.

\title{
Resumen
}

Para definir las características internas y verificar el efecto del almacenamiento sobre los huevos de perdices brasileñas (Rhynchotus rufescens), se evaluaron 50 huevos, distribuidos en un diseño completamente al azar y mantenidos a la temperatura ambiente durante 28 días. Fueron evaluados peso del huevo, altura de albúmina, ancho y altura de yema, unidad Haugh, índice yema, $\mathrm{pH}$ de los componentes internos y color de yema. El color de la yema se determinó mediante el método de caracterización cromática directa [luminosidad (L) y cromaticidad $\left(\mathrm{a}^{*}\right)\left(\mathrm{b}^{*}\right)$ ]. Las evaluaciones se realizaron los días $0,10,15,21$ y 28 inmediatamente después de la puesta. Hubo reducción $(\mathrm{p}<0.05)$ en la altura de la albúmina y en la unidad Haugh, aumento $(\mathrm{p}<0.05)$ en el $\mathrm{pH}$ de la albúmina, disminución $(\mathrm{p}<0.05)$ en la intensidad del rojo $\left(a^{*}\right)$ y aumentar $(\mathrm{p}<0.05)$ en la intensidad del amarillo $\left(\mathrm{b}^{*}\right)$ en la yema. El valor de la unidad Haugh disminuyó de 84.74 a 41.59, la albúmina disminuyó en $4.37 \mathrm{~mm}$ y se volvió más alcalina (pH final 9.58). La yema mostró un color más amarillento, con aumento de 11,51 índice $\mathrm{b}^{*}$ y reducción de $-1,01$ índice $\mathrm{a}^{*}$. Los otros parámetros de calidad de la yema no fueron influenciados ( $p>0.05)$ por el tiempo. Se concluye, por tanto, que los huevos de perdiz brasileña tienen características internas con gran potencial de explotación comercial. Sin embargo, 28 días de almacenamiento a la temperatura ambiente son suficientes para afectar la calidad de la albúmina y el color de la yema.

Palabras clave: Albúmina; Índice de yema; pH; Unidad Haugh; Yema.

\section{Introdução}

A criação racional da perdiz brasileira (Rhynchotus rufescens) tem ganhando notoriedade por sua fácil adaptação ao cativeiro e resultados produtivos promissores. Apesar de ser uma ave em processo de domesticação, apresenta bons índices de desempenho (Moro et al., 2000; Tholon \& Queiroz, 2007), rendimento de carcaça (Moro et al., 2006; Queiroz et al., 2013) e adaptabilidade a novas dietas (Felipe et al., 2010; Moro et al., 2002; Nakage et al., 2002). Sua exploração zootécnica visa a produção de cortes cárneos exóticos, mas em tempos que se buscam fontes alternativas de proteína animal e ovos cada vez mais diferenciados no mercado, é importante que se explore a potencialidade dessa espécie para a produção de ovos. Embora apresente reprodução estacional, as características físicas de seus ovos são bastante peculiares, tornando-os bastante atrativos ao consumidor, com casca de coloração vinácea ao chocolate violáceo e brilhante, assemelhando-se à porcelana (Sick, 1997).

Assim como observado em ovos de outras aves domésticas, espera-se que o avançar do período de armazenamento comprometa sua qualidade (Moura et al., 2008; Scott \& Silversides, 2000), promovendo redução na altura dos componentes internos, alcalinidade e alterações em suas caraterísticas sensoriais. A medida mais utilizada para indicar a qualidade interna do ovo é a unidade Haugh (Alleoni \& Antunes, 2001), quanto maior seu valor, melhor a qualidade do albúmen. O índice de gema também tem sido bastante empregado, visto que seus valores diminuem com o transcorrer do tempo de armazenamento, 
indicando perda na qualidade do ovo ao atingirem índices inferiores a 0,25 (Austic \& Nesheim, 1990). Entre os atributos sensoriais, a cor tem sido bastante utilizada como indicador de qualidade interna (Silva et al., 2000; Tocchini \& Mercadante, 2001). Segundo Moura et al. (2009), a cor da gema é decisória na aquisição do produto pelo consumidor.

As informações que se tem atualmente sobre as características internas dos ovos das perdizes brasileiras são meramente empíricas, baseadas em seu consumo nas propriedades rurais e como excedente da criação das aves voltadas para corte. Dessa forma, o conhecimento básico de suas características internas se faz de fundamental importância, pois permite avaliar o potencial de mercado de um produto diferenciado, com garantida qualidade e segurança alimentar. Portanto, buscouse com o presente estudo caracterizar e avaliar a qualidade interna dos ovos de perdizes brasileiras ao longo de 28 dias de armazenamento.

\section{Metodologia}

O presente estudo foi desenvolvido segundo o método quantitativo experimental, tal qual descrito por Brito et al. (2020), no Laboratório de Análise de Alimentos de Origem Animal da Faculdade de Ciências Agrárias e Veterinárias da UNESP, Jaboticabal, SP. Foram utilizados 50 ovos frescos de perdizes brasileiras Rhynchotus rufescens, em estação reprodutiva e ciclo intermediário de postura, gentilmente cedidos pelo Setor de Recria de Perdizes da supracitada Instituição. As aves foram criadas sob as mesmas condições de manejo, ambiência, alimentação e possuíam em média 36 meses de idade.

Os ovos foram individualmente identificados, pesados, distribuídos em delineamento experimental inteiramente casualizado, armazenados por 28 dias em embalagens plásticas à temperatura ambiente $\left(25,7 \pm 0,9^{\circ} \mathrm{C}\right)$ e analisados em cinco tempos $(0,10,15,21$ e 28 dias após a postura). Em cada tempo foram avaliados 10 ovos, quando foram novamente pesados, quebrados e registradas a altura de albúmen $(\mathrm{mm})$, largura e altura de gema $(\mathrm{mm}), \mathrm{pH}$ dos componentes internos e coloração da gema crua.

As alturas de albúmen denso e gema foram mensuradas com o auxílio de altímetro especial (Egg Quality Micrometer). Com a relação entre o peso do ovo e a altura do albúmen, obteve-se a unidade Haugh por meio da fórmula descrita por Card e Nesheim (1966):

$$
\begin{aligned}
& \mathrm{UH}=100 * \log (\mathrm{H}+7,57-1,7 \mathrm{~W} 0,37) \\
& \text { onde: } \\
& \mathrm{UH}=\text { unidade Haugh; } \\
& \mathrm{H}=\text { altura do albúmen }(\mathrm{mm}) ; \\
& \text { W = peso do ovo }(\mathrm{g}) .
\end{aligned}
$$

Com o auxílio de um paquímetro de alta precisão, mensurou-se a largura da gema. A relação entre altura e largura de gema forneceu o índice de gema:

$$
\mathrm{IG}=\mathrm{AG} / \mathrm{LG}
$$

onde:

IG = índice de gema; 


$$
\begin{aligned}
& \mathrm{AG}=\text { altura da gema }(\mathrm{mm}) \\
& \mathrm{LG}=\text { largura da gema }(\mathrm{mm})
\end{aligned}
$$

A coloração da gema foi verificada por determinação direta da caracterização cromática. Para tal, foi utilizado o colorímetro Minolta CR-400, previamente calibrado em superfície branca de acordo com padrões pré-estabelecidos por Bible e Singha (1993) e Mutschler et al. (1992). Os valores de $a^{*}, b^{*}$ e L foram tomados em triplicata. O valor de $a^{*}$ caracteriza coloração na região do vermelho $\left(+a^{*}\right)$ ao verde $\left(-a^{*}\right)$ e o valor $b^{*}$ indica coloração no intervalo do amarelo (+b*) ao azul $(-$ b*). O valor $\mathrm{L}$ define os valores de luminosidade, variando do preto $(\mathrm{L}=0)$ ao branco $(\mathrm{L}=100)$.

$\mathrm{O}$ pH de gema e albúmen foi determinado por meio de pHmetro digital (Testo 205), após separação e homogeneização do "pool” de cada componente, com introdução direta do eletrodo nas amostras.

Para classificação da qualidade interna dos ovos, foi adotado o sistema preconizado pelo Departamento de Agricultura dos Estados Unidos (USDA, 2000).

A análise de variância dos dados foi realizada pelo programa Statistical Analysis System (SAS, 2002), e as médias comparadas pelo teste Tukey a $5 \%$ de probabilidade.

\section{Resultados e Discussão}

Ao longo do período experimental, verificou-se queda $(\mathrm{p}<0,05)$ nos valores de unidade Haugh e redução $(\mathrm{p}<0,05)$ na altura do albúmen, com concomitante elevação $(\mathrm{p}<0,05)$ de seu $\mathrm{pH}$. A coloração da gema crua também foi influenciada pelo tempo de armazenamento dos ovos, com aumento $(\mathrm{p}<0,05)$ da intensidade de amarelo e redução $(\mathrm{p}<0,05)$ da intensidade de vermelho. Os demais parâmetros avaliados, a saber, índice de gema, pH da gema, largura da gema, peso do ovo e luminosidade, não foram afetados ( $\mathrm{p}>0,05)$ pelo tempo de armazenamento, conforme os dados apresentados na Tabela 1. 
Tabela 1. Médias das variáveis analisadas de acordo com o período de armazenamento dos ovos das perdizes brasileiras.*

Período de armazenamento (dias)

\begin{tabular}{|c|c|c|c|c|c|c|c|}
\hline \multirow{2}{*}{ Variáveis } & \multicolumn{5}{|c|}{ Período de armazenamento (dias) } & \multirow{2}{*}{ Médias } & \multirow{2}{*}{$\mathrm{CV}^{* *}(\%)$} \\
\hline & 0 & 10 & 15 & 21 & 28 & & \\
\hline Unidade Haugh & $84,74^{\mathrm{a}}$ & $70,41^{\mathrm{ab}}$ & $71,13^{\mathrm{ab}}$ & $54,02^{\mathrm{ab}}$ & $41,59^{\mathrm{b}}$ & 66,25 & 24,41 \\
\hline Índice de gema & 0,37 & 0,37 & 0,40 & 0,37 & 0,38 & 0,38 & 16,12 \\
\hline pH albúmen & $8,52^{\mathrm{c}}$ & $9,34^{\mathrm{b}}$ & $9,41^{\mathrm{ab}}$ & $9,47^{\mathrm{ab}}$ & $9,58^{\mathrm{a}}$ & 9,31 & 1,19 \\
\hline $\mathrm{pH}$ gema & 6,24 & 6,61 & 6,88 & 6,70 & 6,43 & 6,63 & 4,79 \\
\hline Luminosidade (L) & 44,79 & 46,14 & 42,65 & 46,07 & 51,20 & 45,60 & 20,93 \\
\hline $\begin{array}{l}\text { Intensidade de vermelho } \\
\qquad\left(\mathrm{a}^{*}\right) \text { da gema }\end{array}$ & $-3,66^{a}$ & $-4,88^{a b}$ & $-4,57^{a b}$ & $-4,32^{a}$ & $-5,83^{b}$ & $-4,67$ & 14,82 \\
\hline $\begin{array}{l}\text { Intensidade de amarelo } \\
\qquad\left(b^{*}\right) \text { da gema }\end{array}$ & $29,89^{b}$ & $35,67^{\mathrm{ab}}$ & $39,83^{\mathrm{a}}$ & $38,36^{\mathrm{a}}$ & $41,40^{\mathrm{a}}$ & 37,25 & 10,86 \\
\hline Largura de gema $(\mathrm{mm})$ & 44,33 & 45,75 & 42,71 & 44,75 & 43,33 & 44,28 & 9,41 \\
\hline Altura de gema (mm) & 16,30 & 16,55 & 17,04 & 16,35 & 16,57 & 16,63 & 9,33 \\
\hline Altura de albúmen (mm) & $7,04^{\mathrm{a}}$ & $5,20^{\mathrm{ab}}$ & $4,93^{\mathrm{ab}}$ & $3,88^{\mathrm{ab}}$ & $2,67^{b}$ & 4,83 & 31,80 \\
\hline Peso do ovo (g) & 56,44 & 58,33 & 50,74 & 56,32 & 50,10 & 54,67 & 10,97 \\
\hline
\end{tabular}

*Médias seguidas de letras diferentes na mesma linha diferem entre si pelo teste de Tukey $(\mathrm{p}<0,05)$.

$* * \mathrm{CV}=$ Coeficiente de variação.

Fonte: Elaborada pelos autores.

Os resultados do presente estudo demonstraram que a qualidade do albúmen dos ovos de perdizes brasileiras diminuiu ao longo dos 28 dias de armazenamento em temperatura ambiente, apresentando padrão de deterioração semelhante àqueles já descritos para ovos de outras espécies domésticas armazenados sob mesmas condições (Barbosa et al., 2008; Diana et al., 2020; Jucá et al., 2011; Lana et al. 2017; Lopes et al., 2012).

O menor valor para unidade Haugh (UH) foi verificado no $28^{\circ}$ dia de armazenamento (Tabela 1), similarmente aos resultados encontrados por Diana et al. (2020), ao registrarem valor mínimo para UH em ovos de galinhas comerciais (Hyline W36) aos 30 e 33 dias de armazenamento. Com o avançar do tempo de armazenamento, o valor médio da unidade Haugh (UH) apresentou redução quadrática de 84,74 para 41,59 , do 0 ao $28^{\circ}$ dia de estocagem, com elevado coeficiente de determinação $\left(\mathrm{R}^{2}=0,89\right)$, demonstrando que não houve dispersão dos dados encontrados.

O Departamento de Agricultura dos Estados Unidos classifica os ovos segundo os valores de UH: AA - ovos de excelente qualidade $(\mathrm{UH} \geq 72), \mathrm{A}-$ ovos de alta qualidade $(71 \geq \mathrm{UH} \geq 60)$ e $\mathrm{B}-$ ovos de baixa qualidade (59 $\geq \mathrm{UH})$. Dessa forma, de acordo com a equação de regressão encontrada ( $\left.Y=83,1947-0,6601 x-0,0294 x^{2}\right)$, assume-se que os ovos das perdizes armazenados em temperatura ambiente podem ser classificados como AA até o $9^{\circ}$ dia após a postura, A entre os dias 10 e 17 e B a partir do $18^{\circ}$ dia de armazenamento. 
Segundo esta equação, os valores de UH diminuíram em média 1,51 a cada dia de armazenamento, representando uma queda de 50,92\% durante todo o período experimental. Valores semelhantes foram registrados por Barbosa et al. (2008), a partir da equação de regressão obtida para ovos de galinha armazenados em condições ambientais não controladas, onde os ovos da linhagem Hisex branca diminuíram 1,82 UH por dia, enquanto os ovos de HyLine branca apresentaram queda de 1,48 UH por dia.

O declínio dos valores de UH esteve diretamente relacionado à diminuição da altura do albúmen. Com o transcorrer do tempo de armazenamento, o albúmen se tornou mais fluido, reduzindo seu tamanho em 62,07\%, o equivalente a uma perda de 4,37 mm, quando comparados os tempos 0 e 28 dias (Tabela 1). Resultados semelhantes foram registrados por Silversides e Villeneuve (1994), ao verificarem que o longo tempo de prateleira proporcionou redução de $77 \%$ na altura do albúmen e $78 \%$ de unidade Haugh em ovos provenientes de poedeiras comerciais.

Essa fluidificação do albúmen, representada pela redução de sua altura, é um indicador da perda de qualidade dos ovos (Sauveur, 1993), que acaba por influenciar as respostas expressas em UH. À medida que o ovo envelhece, o albúmen denso se torna mais líquido, reduzindo os valores de UH (Xavier et al., 2008). De acordo com Moreng e Avens (1990), as enzimas presentes no albúmen hidrolisam cadeias aminoacídicas, que ao destruírem sua estrutura liberam água (antes ligada a grandes moléculas de proteínas) para a gema, promovendo liquefação, perda de viscosidade do albúmen mais denso (Austic \& Nesheim, 1990) e redução de sua altura.

A redução na altura de albúmen dos ovos das perdizes, verificada a partir do $10^{\circ}$ dia de armazenamento (Tabela 1), coincide àquela descrita por Scott e Silversides (2000), os quais registraram altura média de 4,75 $\mathrm{mm}$ em ovos de poedeiras Isa-White e Isa-Brown no $10^{\circ}$ dia de armazenamento em temperatura ambiente. Diana et al. (2020) e Lana et al. (2017) também verificaram diminuição da altura de albúmen ao armazenarem ovos de poedeiras comerciais das linhagens HyLine W36 e Dekalb White, respectivamente. Comportamento semelhante foi observado em ovos de codorna (Moura et al., 2008), com redução da UH da altura de albúmen ao longo do armazenamento em temperatura ambiente.

Com relação aos valores de $\mathrm{pH}$ encontrados para os ovos das perdizes, estes se enquadram dentro dos valores descritos na literatura para ovos de galinha e codorna japonesa (Barbosa et al., 2008; Diana et al., 2020; Moura et al., 2008; Scott \& Silversides, 2000).

Para o pH da gema, não foram observadas diferenças significativas, indicando estabilidade durante os 28 dias de armazenamento, com valor médio de 6,63 (Tabela 1). Sob mesma condições, Diana et al. (2020), ao avaliarem ovos de galinha (HyLine W36) em diferentes embalagens e tempos de armazenamento, registraram valores extremos do $\mathrm{pH}$ de gema somente aos 35 dias de armazenamento (pH: 6,43).

Já o $\mathrm{pH}$ do albúmen, aos 28 dias ( $\mathrm{pH}: 9,58$ ) (Tabela 1), assumiu valores significativamente superiores àqueles encontrados em ovos com 0 e 10 dias de armazenamento ( $\mathrm{pH}$ : 8,52 e 9,34; respectivamente) e similares àqueles encontrados aos 15 e 21 dias de armazenamento (pH: 9,41 e 9,47; respectivamente).

Em ovos frescos de aves domésticas, o pH do albúmen apresenta valores entre 7,6 e 8,54, alcançando média elevadas como 9,64 após alguns dias de armazenamento em temperatura ambiente (Akyurek \& Okur, 2009; Coutts, Wilson, \& Fernandez, 2007; Xavier et al., 2008), tal qual observado no presente trabalho. Resultados similares foram registrados por Diana et al. (2020), os quais verificaram maior pH no albúmen de ovos de galinha (HyLine W36) ao $28^{\circ}$ dia de armazenamento em embalagens plásticas (pH: 9,69). Jucá et al. (2011) também observaram elevação no pH do albúmen de ovos de poedeiras Isa-Brown criadas em sistema sem ventilação, após 30 dias de armazenamento (dia 0: 8,60; dia 30: 9,70).

$\mathrm{O}$ aumento no $\mathrm{pH}$ do albúmen, observado durante o armazenamento dos ovos das perdizes, está intimamente ligado à 
perda de dióxido de carbono para o meio externo, através dos poros presentes na casca. Com essa perda, ocorre o desequilíbrio no sistema tampão do ácido carbônico (H2CO3) e consequente elevação no pH do albúmen. Com o meio alcalinizado, ocorre a dissociação química do complexo proteico, originando a ruptura da estrutura em gel do albúmen denso (Fennema, 1993), como pode ser inferido ao se correlacionar o mais elevado $\mathrm{pH}$ de albúmen encontrado $(\mathrm{pH}$ : 9,58) à sua redução em tamanho $(-4,3$ $\mathrm{mm}$ ), ambos observados aos 28 dias de armazenamento (Tabela 1).

Baseando-se nos valores do índice de gema (IG), constatou-se que os ovos de perdizes se mantiveram estáveis ao longo dos 28 dias de armazenamento, apresentando valor médio aceitável para consumo (IG: 0,38) (Tabela 1). Valores de IG para ovos frescos são considerados ideais quando dentro do intervalo 0,35 a 0,49 (Santos, 2008), enquanto valores inferiores a 0,25 caracterizariam gema muito frágil e de difícil mensuração (Card \& Nesheim, 1978), portanto, de baixa qualidade. Dados similares foram observados por Leonel et al. (2007), ao estudarem ovos armazenados em filme PVC e a vácuo, obtendo valor médio de $\mathrm{IG}=0,35$, após 28 dias de armazenamento.

A coloração da gema crua também foi influenciada pelo tempo de armazenamento dos ovos, com exceção à luminosidade. A intensidade de amarelo aumentou significativamente a partir do $15^{\circ}$ dia experimental (Tabela 1), com valores superiores ao dia 0 e semelhantes ao dia 10; enquanto a intensidade de vermelho foi estatisticamente inferior no $28^{\circ}$ dia em relação aos demais dias analisados.

Com tonalidade mais amarelada, expressa pelos valores da coordenada $b^{*}$, a pigmentação da gema variou de 29,89 a 41,40 (+11,51) durante o período avaliado (Tabela 1), corroborando os dados obtidos por Harder, Canniatti-Brazaca e Arthur (2007). Freitas et al. (2011) também observaram intensidade de amarelo crescente $(38,15$ a 40,41) em gemas cruas de ovos de poedeiras Isa-Brown, entre o $7^{\circ}$ e $21^{\circ}$ dia de armazenamento. Resultados semelhantes ainda foram registrados por Moura et al. (2010), ao verificaram índice b* médio de 30,17 em gema de ovos de codorna japonesa no dia da postura.

A coloração amarelada da gema dos ovos das perdizes se deu como resultado direto de sua alimentação à base de milho e farelo de soja. Esses alimentos são ricos em pigmentos carotenoides amarelos, conhecidos como xantofilas, e sua presença na dieta das aves determina a cor da gema dos ovos (Awang, Chulan \& Ahmad, 1992; Hencken, 1992), especialmente pela ação da luteína e zeaxantina, principais xantofilas presentes no milho (Biscaro \& Canniatti-Brazaca, 2006). Quanto maior o teor desses pigmentos na dieta, maior será sua deposição nas gemas e a intensidade de coloração (Bhosale \& Bernstein, 2005; Ponsano et al., 2004). Esses carotenoides acumulam-se na porção lipídica das lipoproteínas do ovo, conferindo pigmentação de gema na faixa do amarelo (Jacob, Biles \& Mather, 2000).

Já a cromaticidade $\mathrm{a}^{*}$ foi negativa (Tabela 1), indicando total ausência de coloração vermelha na gema, e revelou um aumento na intensidade de verde ao longo do tempo de armazenamento, com índices de reflectância compreendidos na faixa do verde (-a*) entre $-3,66$ e - 5,83 (- 2,17), diferentemente dos resultados na faixa do positivo já encontrados para gema de outras espécies comerciais (Brito et al., 2020; Harder et al., 2007). Harder et al. (2007), estudando a intensidade da coloração da gema de ovos de galinhas poedeiras alimentadas com urucum (Bixa orellana), obtiveram valores de cromaticidade ( $\mathrm{a}^{*}$ ) variando de 2,23 $\pm 3,25$ a 9,61 $\pm 1,69$. Isso demonstra que a coloração esverdeada da gema pode ser atribuída à alimentação, mas também às características intrínsecas da espécie estudada, o que demanda mais estudos sobre o tema.

O estabelecimento de tonalidades mais suaves da gema com o transcorrer do armazenamento, foi, provavelmente, ocasionado pela transferência do ferro da gema para o albúmen, ao longo do tempo, promovendo sua despigmentação (Sauveur, 1993) e consequente contribuição para o estabelecimento dos valores de intensidade luminosa (L: 45,60). 


\section{Conclusões}

A gema dos ovos frescos das perdizes brasileiras apresenta uma coloração clara e suave, com tons amareloesverdeados que se intensificam ao longo do período de armazenamento, apresentando características internas compatíveis aos ovos de outras aves domésticas já introduzidas no mercado avícola, caracterizando um produto de excelente qualidade e com grande potencial de exploração comercial. Entretanto, 28 dias de armazenamento em temperatura ambiente é suficiente para afetar a qualidade do albúmen e a coloração da gema.

Esses resultados compreendem os primeiros achados relacionados à qualidade interna dos ovos de perdizes brasileiras (Rhynchotus rufescens) voltados para o consumo humano, haja visto que estudos recentes envolvendo estes ovos têm seu foco na qualidade de incubação, e não no seu potencial enquanto alimento. O que, por sua vez, traz à luz novas e promissoras possibilidades de pesquisas científicas, de forma a incentivar e propagar a criação racional desta espécie, como fonte alternativa de proteína, quer seja por sua aptidão para carne, quer seja por sua produção de ovos exóticos.

\section{Referências}

Akyurek, H.; \& Okur, A. G. (2009). Effect of storage time, temperature and hen age on egg quality in free range layer hens. Journal of Animal and Veterinary, $8,1953-1958$

Alleoni, A. C. C., \& Antunes, A. J. (2001). Unidade Haugh como medida da qualidade de ovos de galinha armazenados sob refrigeração. Scientia Agrícola, $58,681-685$.

Austic, R. E., \& Nesheim, M. C. (1990). Poultry production. (13a ed). Philadelphia, Estados Unidos: Lea \& Febiger.

Awang, I. P. R., Chulan, U., \& Ahmad, F. B. H. (1992). Curcumin for upgrading skin color of broilers. Pertanika, 15(1), 37-38.

Barbosa, N. A. A., Sakomura, N. K., Mendonça, M. O., Freitas, E. R., \& Fernandes, J. B. K. (2008). Qualidade de ovos comerciais provenientes de poedeiras comerciais armazenados sob diferentes tempos e condições de ambientes. Ars Veterinária, v.24, n.2, p.127-133.

Bible, B. B., \& Singha, S. (1993). Canopy position influences CIELAB coordinates of peach color. HortScience, $28(10), 992$ - 993.

Biscaro, L. M., \& Caniatti-Brazaca, S. G. (2006). Cor, betacaroteno e colesterol em gema de ovos obtidos de poedeiras que receberam diferentes dietas. Ciência Agrotécnica, 6, 1130-1134.

Bhosale, P., \& Bernstein, P. S. (2005). Microbial xanthophylls. Applied Microbiology Biotecnology, 68(4), $445-455$.

Brito, D. R. N., Pereira, A. L. F., Mota, A. S. B., Freitas, E. R., \& Abreu, V. K. G. (2020). Eggs quality sold at different establishments in the Imperatriz city, Maranhão State, Brazil. Research, Society and Development, 9(9), e749997848.

Card, L. E., \& Neisheim, M. C. (1966). Poultry production. Philadelphia: Lea \& Febiger.

Card, L. E., \& Nesheim, M. C. (1978). Producción Avícola. Zaragoza: Editoral Acribia.

Coutts, J. A., Wilson, G. C., \& Fernandez, S. (2007). Optimum egg quality - A practical approach. Sheffield: 5M Enterprises.

Diana, T. F., Cobucci, J. M., Marques, K. C., Teixeira, A. O., Brighenti, C. R. G., Ferreira, V. P. A., \& Reis, R. S. (2020). Qualidade de ovos acondicionados em diferentes tipos de embalagens e armazenados em postos de comercialização no Município de São João del - Rei, MG. Research, Society and Development, 9(9), e328997337.

Felipe, L., Santos, E. C., Tavian, A. F., Góes, P. A. A., Moraes, V. M. B., Tonhati, H., ... Queiroz, S. A. (2010). Effect of crude protein levels and organic selenium supplementation in the diets fed during the breeding season on reproductive parameters of red-winged tinamous (Rhynchotus rufescens). Brazilian Journal of Poultry Science, 12(1), $63-71$.

Fennema, O. R. (1993). Química de los alimentos. (2a ed.), Zaragoga: Acribia.

Freitas, L. W., Paz, I. C. L., Garcia, R. G., Caldara, F. R., Seno, L. O.; Feliz, G. A.; ... Cavichiolo, F. (2011). Aspectos qualitativos de ovoscomerciais submetidos a diferentes condições de armazenamento. Revista Agrarian, 4(11), 66-72.

Harder, M. N. C., Canniatti-Brazaca, S. G., \& Arthur, V. (2007). Avaliação quantitativa por colorímetro digital da cor do ovo de galinhas poedeiras alimentadas com urucum (Bixa orellana). Revista Portuguesa de Ciências Veterinárias, 102, 339-342.

Haugh, R. R. (1937). The Haugh unit for measuring egg quality. United States Egg Poultry Magazine, 43, 552 - 555.

Hencken, H. (1992). Chemical and physiological behavior of feed carotenoids and their effects on pigmentation. Poultry Science, 71(4), 711-717. 
Jacob, J. P., Miles, R. D., \& Mather, F. B. (2011). Egg quality. Flórida: Universidade da Flórida.

Jucá, T. S., Gomes, F. A., Silva, L. A., Silva, R. P. M., \& Vale, M. A. D. (2011). Efeito do tempo e condições de armazenamento sobre a qualidade interna de ovos de poedeiras Isa Brown produzidos em diferentes sistemas de criação e ambiência. Enciclopédia Biosfera, 7(13), 446-460.

Lana, S. R. V., Lana, G. R. Q., Salvador, E. L., Lana, Â. M. Q., Cunha, F. S. A.; Marinho, A. L. (2017). Qualidade de ovos de poedeiras comerciais armazenados em diferentes temperaturas e períodos de estocagem. Revista Brasileira de Saúde Produção Animal, 18, 140-151.

Lopes, L. L. R. A., Silva, Y. L., Nunes, R. V., Takahashi, S. E., Mori, C. (2012). Influência do tempo e das condições de armazenamento na qualidade de ovos comerciais. Revista Científica Eletrônica de Medicina Veterinária, 9(18).

Moreng, R. E., \& Avens, J. S. (1990). Ciência e produção de aves (N. M. K. Ito, Trad.). São Paulo: Roca, p. 43-75.

Moro, M. E. G., Ariki, J., \& Malheiros, E. B. (2002). Avaliação dos níveis de proteína da dieta sobre a idade à maturidade sexual e produção de ovos de perdiz (Rhynchotus rufescens, Temminek). Acta Scientiarum, 24, 997 - 1000.

Moro, M. E. G., Arik, J., Souza, P. A., Souza, H. B. A., Moraes, V. M. B., \& Vargas, F. C. (2006). Rendimento de carcaça e composição química da carne da perdiz nativa (Rhynchotus rufescens). Ciência Rural, 36(1), $258-262$.

Moro, M. E. G., Tavares, F. A., \& Lima, C. G. (2000). Desempenho produtivo da perdiz (Rhynchotus rufescens) submetida a rações com diferentes níveis energéticos. Brazilian Journal of Poultry Science, 2(1), 13 - 18.

Moura, A. M. A., Fonseca, J. B., Melo, E. A., Lima, V. L. A. G., Santos, P. A., \& Silva, Q. J. (2009). Características sensoriais de ovos de codornas japonesas (Coturnix japonica temminck e schlegel, 1849) suplementadas com pigmentantes sintéticos e selenometionina. Ciência e Agrotecnologia, $33(6)$, 1594 - 1600.

Moura, A. M. A., Fonseca, J. B., Rabello, A. B. V., Takata, F. N., \& Oliveira, N. T. E. (2010). Desempenho e qualidade do ovo de codornas japonesas alimentadas com rações contendo sorgo. Revista Brasileira de Zootecnia, 39, 2697-2702.

Moura, A. M. A., Oliveira, N. T. E., Thiebaut, J. T. L., \& Melo, T. V. (2008). Efeito da temperatura de estocagem e do tipo de embalagem sobre a qualidade interna de ovos de codornas japonesas (Coturnix japonica). Revista Ciência e Agrotecnologia, 32, 578 - 583.

Mutschler, M. A., Wolfe, D. W., Cobb, E. D., \& Yourstone, K. S. (1992). Tomato fruit-qulity and shelf-life in hybrids heterozygous for the alc ripening mutant. HortScience, Alexandria, 27(4), 352 - 355.

Nakage, E. S., Cardozo, J. P, Pereira, G. T., Queiroz, S. A., \& Boleli, I. C. (2002). Efeito da Forma Física da Ração Sobre a Porosidade, Espessura da Casca, Perda de Água e Eclodibilidade em Ovos de Perdiz (Rhynchotus Rufescens). Brazilian Journal of Poultry Science, $4(3), 227$ - 234.

Ponsano, E. H. G., Pinto, M. F., Neto, M., Garcia, \& Lacava, P. M. (2004). Rhodocyclus gelatinosus biomass for egg yolk pigmentation. Journal of Applied Poultry Research, 3(3), 421-425.

Queiroz, F. A., Carvalho, M. M., Sugui, J. K., Nunes, J., Felipe, L., Santos, E. C.; ... Queiroz, S.A. (2013). Meat and carcass traits of the red-winged tinamou (Rhynchotus rufescens). Brazilian Journal of Poultry Science, 15(2), 113 - 118.

SAS. Statistical Analysis System. (2002). User's guide: statistics 9.0. Cary, NC: SAS Institute Inc.

Santos, A. L. (2008). Desempenho, crescimento, qualidade do ovo, composição centesimal corporal e características reprodutivas e ósseas de poedeiras submetidas a diferentes programas nutricionais (Tese de Doutorado). Universidade de São Paulo, Pirassununga, SP, Brasil.

Sauveur, B. (1993). El huevo para consumo: bases productivas (C. B. Carbó, Trad.). Barcelona: Aedos Editorial.

Scott, T. A., \& Silversides, F. G. (2000). The effect of storage strain of hen on egg quality. Poultry Science, 79(12), 1725 - 1729.

Sick, H. (1997). Ornitologia brasileira, uma introdução. Rio de Janeiro: Editora Nova Fronteira.

Silva, D. J., \& Queiroz, A. C. (2002). Análise de alimentos: métodos químicos e biológicos (3a ed.). Viçosa: UFV.

Silversides, F. G., \& Villeneuve, P. (1994). Is the Unit Haugh correction for egg weight valid for eggs stored at room temperature? Poultry Science, 73(1), 50 -55 .

Tocchini, L., \& Mercadante, A. Z. (2001). Extração e determinação, por CLAE, de bixina e norbixina em coloríficos. Ciência e Tecnologia de Alimentos, $21(3), 310-313$

Tholon, P., \& Queiroz, S.A. (2007). Models for the analysis of growth curves for rearing tinamou (Rhynchotus rufescens) in captivity. Brazilian Journal of Poultry Science, 9(1), 23-31.

USDA. (2000). Egg-Grading Manual. Washington: Departament of Agriculture.

Xavier, I. M. C., Cançado, S. V., Figueiredo, T.C.; Lara, L., Lana, A. M. Q.; Souza, M. R., \& Baião, N. C. (2008). Qualidade de ovos de consumo submetidos a diferentes condições de armazenamento. Arquivo Brasileiro de Medicina Veterinária e Zootecnia, 60(4), 953-959. 